\title{
Role of MSX1 in Osteogenic Differentiation of Human Dental Pulp Stem Cells
}

\author{
Noriko Goto, ${ }^{1}$ Katsumi Fujimoto, ${ }^{2,3}$ Sakiko Fujii, ${ }^{4}$ Hiroko Ida-Yonemochi, ${ }^{5}$ \\ Hayato Ohshima, ${ }^{5}$ Takeshi Kawamoto, ${ }^{2,3}$ Mitsuhide Noshiro, ${ }^{2,3}$ Chisa Shukunami, ${ }^{3}$ \\ Katsuyuki Kozai, ${ }^{1}$ and Yukio Kato ${ }^{2}$
}

${ }^{1}$ Department of Pediatric Dentistry, Institute of Biomedical \& Health Sciences, Hiroshima University, 1-2-3 Kasumi, Minami-ku, Hiroshima 734-8553, Japan

${ }^{2}$ Department of Dental and Medical Biochemistry, Institute of Biomedical \& Health Sciences, Hiroshima University, 1-2-3 Kasumi, Minami-ku, Hiroshima 734-8553, Japan

${ }^{3}$ Department of Molecular Biology and Biochemistry, Institute of Biomedical \& Health Sciences, Hiroshima University, 1-2-3 Kasumi, Minami-ku, Hiroshima 734-8553, Japan

${ }^{4}$ Department of Dental Science for Health Promotion, Institute of Biomedical \& Health Sciences, Hiroshima University, 1-2-3 Kasumi, Minami-ku, Hiroshima 734-8553, Japan

${ }^{5}$ Division of Anatomy and Cell Biology of the Hard Tissue, Department of Tissue Regeneration and Reconstruction, Niigata University Graduate School of Medical and Dental Sciences, 2-5274 Gakkocho-dori, Chuo-ku, Niigata 951-8514, Japan

Correspondence should be addressed to Katsumi Fujimoto; kfujimo@hiroshima-u.ac.jp and Yukio Kato; ykato@hiroshima-u.ac.jp

Received 22 March 2016; Revised 8 July 2016; Accepted 14 July 2016

Academic Editor: Gary E. Lyons

Copyright (c) 2016 Noriko Goto et al. This is an open access article distributed under the Creative Commons Attribution License, which permits unrestricted use, distribution, and reproduction in any medium, provided the original work is properly cited.

Msh homeobox 1 (MSX1) encodes a transcription factor implicated in embryonic development of limbs and craniofacial tissues including bone and teeth. Although MSX1 regulates osteoblast differentiation in the cranial bone of young animal, little is known about the contribution of MSX1 to the osteogenic potential of human cells. In the present study, we investigate the role of MSX1 in osteogenic differentiation of human dental pulp stem cells isolated from deciduous teeth. When these cells were exposed to osteogenesis-induction medium, runt-related transcription factor-2 (RUNX2), bone morphogenetic protein-2 (BMP2), alkaline phosphatase $(A L P L)$, and osteocalcin $(O C N)$ mRNA levels, as well as alkaline phosphatase activity, increased on days 4-12, and thereafter the matrix was calcified on day 14 . However, knockdown of MSX1 with small interfering RNA abolished the induction of the osteoblast-related gene expression, alkaline phosphatase activity, and calcification. Interestingly, DNA microarray and PCR analyses revealed that MSX1 knockdown induced the sterol regulatory element-binding protein 2 (SREBP2) transcriptional factor and its downstream target genes in the cholesterol synthesis pathway. Inhibition of cholesterol synthesis enhances osteoblast differentiation of various mesenchymal cells. Thus, MSX1 may downregulate the cholesterol synthesis-related genes to ensure osteoblast differentiation of human dental pulp stem cells.

\section{Introduction}

Msh homeobox 1 (MSX1) is a homeobox transcriptional factor involved in limb-pattern formation and craniofacial development and specifically in odontogenesis. Mouse Msxi mutations cause craniofacial malformation and tooth agenesis [1]. Msx1-knockout mice show arrested tooth development at the bud stage and embryonic lethal defects [2]. Msxl is expressed at high levels in craniofacial skeletal cells during early postnatal development [3], and transgenic mice expressing Msxl under the control of the alpha (I) collagen promoter exhibit increased osteoblast number, cell proliferation, and apoptosis [4], suggesting Msxl may have a role in craniofacial bone modeling. MSX1 is also expressed at high levels in the dental mesenchyme at the cap and bell stages [5] and may be a suppressor for cell differentiation that maintains mesenchymal cells in a proliferative state to ensure robust craniofacial and tooth development [6]. 
In addition, MSX1 is an upstream and downstream regulator for the bone morphogenetic protein BMP2/BMP4 signaling pathway $[7,8]$. Mutations in human MSX1 also cause cleft lip/palate and tooth agenesis $[9,10]$. However, the role of MSX1 in human craniofacial and tooth development has not been fully understood.

Dental pulp stromal cells isolated from whole pulp tissue can differentiate into osteoblasts, odontoblasts, endothelial cells, nerve cells, and adipocytes in vitro. Some of these cells identified by several cell surface antigens are referred to as dental pulp stem cells (DPSCs) [11, 12]. DPSCs may play a role in dentinogenesis/osteogenesis in both developing and injured teeth. Furthermore, these cells are a promising source of cell-based regenerative therapies for dental, skeletal, vascular, and neuronal diseases $[13,14]$. Human DPSCs (hDPSCs) have not been fully characterized at the molecular level, but a previous reported showed that $M S X 1$ is expressed at higher levels in hDPSCs than in bone marrow-derived mesenchymal stem cells and fibroblasts [15]. MSX1 may participate in the control of primary or secondary dentin formation and reparative dentin or osteodentin/bone formation in injured pulp tissue, in addition to the physiological role such as the maintenance of dental pulp stem/progenitor cells in healthy teeth. In the present study, we explored the role of MSX1 in pulpal mesenchymal cells using human DPSCs in culture.

Statins are a class of drugs that function as specific inhibitors of 3-hydoroxy-3-methylglutaryl-CoA (HMGCoA) reductase, a rate-limiting enzyme in cholesterol synthesis. Numerous studies have shown that statins exert bone anabolic effects in osteoblasts and osteogenic precursor cells $[16,17]$. Simvastatin enhances alveolar bone remodeling in the tooth extraction socket [18], enhances bone fracture healing [19], and reduces alveolar bone loss and tooth mobility in chronic periodontitis [20]. In addition, simvastatin enhances odontoblast/osteoblast differentiation of DPSCs and mesenchymal stem cells isolated from other tissues [17, $21,22]$. These studies indicate a close relationship between cholesterol synthesis and osteoblast differentiation.

Here, we demonstrated the role of MSX1 in osteoblast differentiation and cholesterol synthesis in hDPSCs using small interfering RNA (siRNA) against MSX1. DNA microarray analyses revealed that knockdown of MSX1 in hDPSCs undergoing osteogenic differentiation abolished the expression of various osteoblast-related genes but enhanced the expression of cholesterol synthesis-related genes. Our results suggest that MSX1 enhances osteoblast differentiation and calcification in hDPSCs through repression of cholesterol synthesis genes and induction of osteoblast-related genes.

\section{Material and Methods}

2.1. Human DPSCs. Extracted healthy deciduous teeth were collected from 6-12-year-old children following protocols approved by the ethical authorities at Hiroshima University (permit number: D88-2). Written informed consent was obtained from the subject or subject's parent. Pulp tissue specimens from deciduous teeth were minced and digested with $3 \mathrm{mg} / \mathrm{mL}$ collagenase type I (Life Technologies,
Carlsbad, CA, USA) and $4 \mathrm{mg} / \mathrm{mL}$ dispase (Roche Diagnostics, Mannheim, Germany) in Dulbecco's modified Eagle's medium (DMEM; Sigma, St. Louis, MO, USA) for $1 \mathrm{~h}$ at $37^{\circ} \mathrm{C}$. Single cell suspension was obtained by passing cells through a $70 \mu \mathrm{m}$ cell strainer (CORNING, Corning, NY, USA). The cells were incubated in DMEM supplemented with $20 \%$ fetal bovine serum (FBS; Biowest, Nuaillé, France) and $1 \%$ penicillin-streptomycin (Life Technologies) at $37^{\circ} \mathrm{C}$ in 95\% air and $5 \% \mathrm{CO}_{2}$ [23]. Forming colonies were separated by incubation with Accutase (Funakoshi Co., Ltd., Tokyo, Japan), and isolated cells were transferred to passage cultures with DMEM supplemented by $10 \%$ FBS and $1 \%$ penicillinstreptomycin. The culture medium was changed every 2 days. Cells at passages 3-9 were used in subsequent experiments.

2.2. FACS Analysis. Cells were harvested with Accutase and fixed in $4 \%$ paraformaldehyde. Cells were centrifuged at $1,500 \times \mathrm{g}$ for $5 \mathrm{~min}$ and resuspended at $5 \times 10^{6}$ cells $/ \mathrm{mL}$ in PBS containing $0.5 \%$ bovine serum albumin (BSA). Aliquots containing $10^{5}$ cells were incubated with individual phycoerythrin- (PE-) conjugated antibodies or isotype control PE-conjugated IgG $\kappa$ for $30 \mathrm{~min}$ at room temperature and then washed in PBS supplemented with 3\% FBS. Samples were analyzed using a FACS Aria flow cytometer (Becton Dickinson, Franklin Lakes, NJ, USA) and the data were analyzed using CELLQUEST software (Becton Dickinson). The following monoclonal antibodies were used: PE-conjugated antibodies against CD73 (mouse IgG1 $\kappa$; Biolegend, San Diego, CA, USA), CD90 (mouse IgG1 $\kappa$; Biolegend), CD105

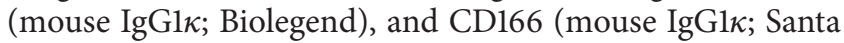
Cruz Biotechnology, Texas, USA). PE-conjugated isotype control mouse IgGl $\kappa$ (Biolegend) was used as the control.

2.3. MSX1 Knockdown. MSX1 siRNA oligonucleotides (s8999 and s224066) were purchased from Life Technologies. The sequences are $5^{\prime}$-GCAUUUAGAUCUACACUCUtt- $3^{\prime}$ (sense) and $5^{\prime}$-AGAGUGUAGAUCUAAAUGCta- $3^{\prime}$ (antisense) for s8999 and $5^{\prime}$-GCAAGA AAAGCGCAGAGAAtt- $3^{\prime}$ (sense) and $5^{\prime}$-UUCUCUGCGCUUUUCUUGCct-3' (antisense) for s224066. Silencer select negative control \#1 siRNA (Life Technologies) was used as the control.

Human DPSCs were seeded at $5 \times 10^{4}$ cells/well in 24 multiwell plates coated with type I collagen with $0.5 \mathrm{~mL}$ DMEM supplemented with $10 \%$ FBS. After $24 \mathrm{~h}$, siRNA was transfected into cells with Lipofectamine 2000 (Life Technologies) and cells were incubated for an additional $48 \mathrm{~h}$.

2.4. Osteogenic Differentiation of hDPSCs and Alizarin Red Staining. After the cultures became confluent, hDPSCs were incubated with $0.5 \mathrm{~mL}$ of DMEM supplemented with $10 \%$ FBS, $10 \mathrm{mM} \beta$-glycerophosphate (Tokyo Chemical Industry Co., Ltd., Tokyo, Japan), $50 \mu \mathrm{g} / \mathrm{mL}$ ascorbic acid 2-phosphate (Sigma), $2 \mathrm{mM}$ L-glutamine (Sigma), $100 \mathrm{nM}$ dexamethasone (Sigma), and 1\% penicillin/streptomycin (osteogenesisinduction medium) as described [15]. For evaluation of calcification, cells incubated with osteogenesis-induction medium for 14 days were fixed at room temperature in $95 \%$ ethanol for 
TABLE 1: Primer and probe sequences used for RT-qPCR.

\begin{tabular}{|c|c|c|}
\hline Gene & Primer $\left(5^{\prime} \rightarrow 3^{\prime}\right)$ & Probe \\
\hline \multirow{2}{*}{ MSX1 (sense) } & F: CTCGTCAAAGCCGAGAGC & \multirow{2}{*}{ Roche Universal Probe \# 7} \\
\hline & R: CGGTTCGTCTTGTGTTTGC & \\
\hline \multirow{2}{*}{ MSX1 (antisense) } & F: GCCAGCCCTCTTAGAAACAG & \multirow{2}{*}{ Roche Universal Probe \# 50} \\
\hline & R: AATAAAGCAGCCCCTCGTTC & \\
\hline \multirow{2}{*}{ RUNX2 } & F: CAGTGACACCATGTCAGCAA & \multirow{2}{*}{ Roche Universal Probe \# 66} \\
\hline & R: GCTCACGTCGCTCATTTTG & \\
\hline \multirow{2}{*}{ BMP2 } & F: CGGACTGCGGTCTCCTAA & \multirow{2}{*}{ Roche Universal Probe \# 49} \\
\hline & R: GGAAGCAGCAACGCTAGAAG & \\
\hline \multirow{2}{*}{ OSX } & F: CAGCAGCTAAACTTGGAAGGA & \multirow{2}{*}{ Roche Universal Probe \# 76} \\
\hline & R: TGCTTTCGCTTGTCTGAGTC & \\
\hline \multirow{2}{*}{$\mathrm{OCN}$} & F: GCCTCCTGAAAGCCGATGT & \multirow{2}{*}{$5^{\prime}$-CCAACTCGTCACAGTCCGGATTGAGCT-3' } \\
\hline & R: AAGAGACCCAGGCGCTACCT & \\
\hline \multirow{2}{*}{ ALPL } & F: TCACTCTCCGAGATGGTGGT & \multirow{2}{*}{ Roche Universal Probe \# 12} \\
\hline & R: GTGCCCGTGGTCAATTCT & \\
\hline \multirow{2}{*}{ SOX9 } & F: GTACCCGCACTTGCACAAC & \multirow{2}{*}{ Roche Universal Probe \# 61} \\
\hline & R: TCTCGCTCTCGTTCAGAAGTC & \\
\hline \multirow{2}{*}{$\operatorname{PPAR} \gamma$} & F: GACAGGAAAGACAACAGACAAATC & \multirow{2}{*}{ Roche Universal Probe \# 7} \\
\hline & R: GGGGTGATGTGTTTGAACTTG & \\
\hline \multirow{2}{*}{ SREBP2 } & F: GCCCTGGAAGTGACAGAGAG & \multirow{2}{*}{ Roche Universal Probe \# 21} \\
\hline & R: TGCTTTCCCAGGGAGTGA & \\
\hline \multirow{2}{*}{ HMGCS1 } & F: TCTGTCTACTGCAAAAAGATCCAT & \multirow{2}{*}{ Roche Universal Probe \# 59} \\
\hline & R: TGAAGCCAAAATCATTCAAGG & \\
\hline \multirow{2}{*}{ HMGCR } & F: GTTCGGTGGCCTCTAGTGAG & \multirow{2}{*}{ Roche Universal Probe \# 65} \\
\hline & R: GCATTCGAAAAAGTCTTGACAAC & \\
\hline \multirow{2}{*}{ FDPS } & F: GGCCACTCCAGAACAGTACC & \multirow{2}{*}{ Roche Universal Probe \# 75} \\
\hline & R: CCTCATATAGCGCCTTCACC & \\
\hline \multirow{2}{*}{ CYP51A1 } & F: TGCAGATTTGGATGGAGGTT & \multirow{2}{*}{ Roche Universal Probe \# 64} \\
\hline & R: CCTTGATTTCCCGATGAGC & \\
\hline \multirow{2}{*}{ DHCR7 } & F: GCCATGGTCAAGGGCTAC & \multirow{2}{*}{ Roche Universal Probe \# 60} \\
\hline & R: TTGTAAAAGAAATTGCCTGTGAAT & \\
\hline
\end{tabular}

MSX1: msh homeobox 1; RUNX2: runt-related transcription factor-2; BMP2: bone morphogenetic protein-2; OSX: osterix; OCN: osteocalcin; ALPL: alkaline phosphatase liver type; SOX9: SRY- (sex determining region Y-) box 9; PPAR $\gamma$ : peroxisome proliferator activated receptor gamma; SREBP2: sterol regulatory element-binding protein 2; HMGCS1: 3-hydroxy-3-methylglutaryl-CoA synthase 1; HMGCR: HMG-CoA reductase; FDPS: farnesyl diphosphate synthase; CYP51A1: Cytochrome P450 Family 51 Subfamily A Polypeptide 1; DHCR7: 7-dehydrocholesterol reductase; F: forward; R: reverse.

$10 \mathrm{~min}$ and stained with $1 \%$ alizarin red $\mathrm{S}$ for $30 \mathrm{~min}$. The cellmatrix layers were washed 6 times with sterile water.

2.5. Alkaline Phosphatase Activity. Human DPSCs were washed twice with saline and homogenized ultrasonically with 1\% NP-40 in saline. Alkaline phosphatase activity was determined using Lab Assay ALP (Wako, Osaka, Japan). DNA concentration was determined with the Quant-iT ${ }^{\mathrm{TM}}$ PicoGreen dsDNA Assay Kit (Life Technologies) to calculate alkaline phosphatase activity/ $\mu \mathrm{g}$ DNA.

2.6. Reverse Transcription-Quantitative Polymerase Chain Reaction (RT-qPCR). Total RNA was isolated and cDNA was synthesized as described [15]. The cDNA samples were amplified using Universal PCR Master Mix (Life Technologies) with primers (Table 1) and TaqMan probes were purchased from Roche Diagnostics (Basel, Switzerland). GAPDH primers/probe set was used for normalization. After amplification of DNA, expression levels were determined with the ABI prism $7900 \mathrm{HT}$ sequence detection system (Life Technologies).

2.7. DNA Microarray. After the induction of osteogenic differentiation for 4 days, total RNA was isolated from MSX1-knockdown and control hDPSCs using TRIzol (Life Technologies, Japan) and an RNeasy Mini Kit (Qiagen, Chatsworth, CA). DNA microarray analysis was performed using the SurePrint G3 Human GE $8 \times 60 \mathrm{~K}$ v2 Microarray (Agilent Technologies, Santa Clara, CA, USA). Raw data were standardized by the global median normalization method using GeneSpring (Silicon Genetics, Redwood City, CA, USA). The raw data were deposited in the Gene Expression Omnibus database (GSE69992).

2.8. Statistical Analysis. Results are expressed as mean \pm SD. Differences between two groups were analyzed by two-way 


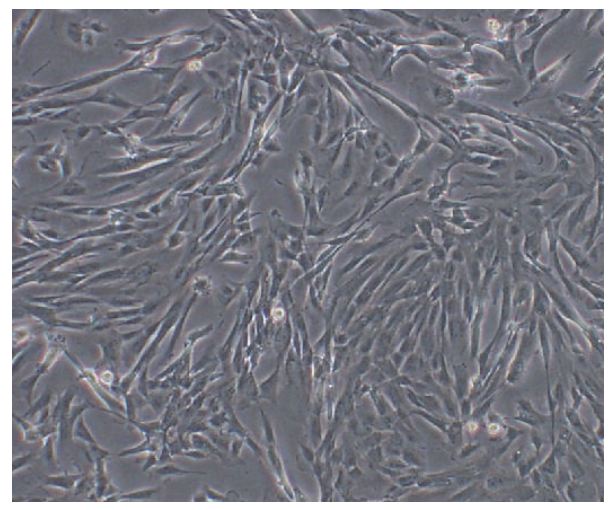

(a)
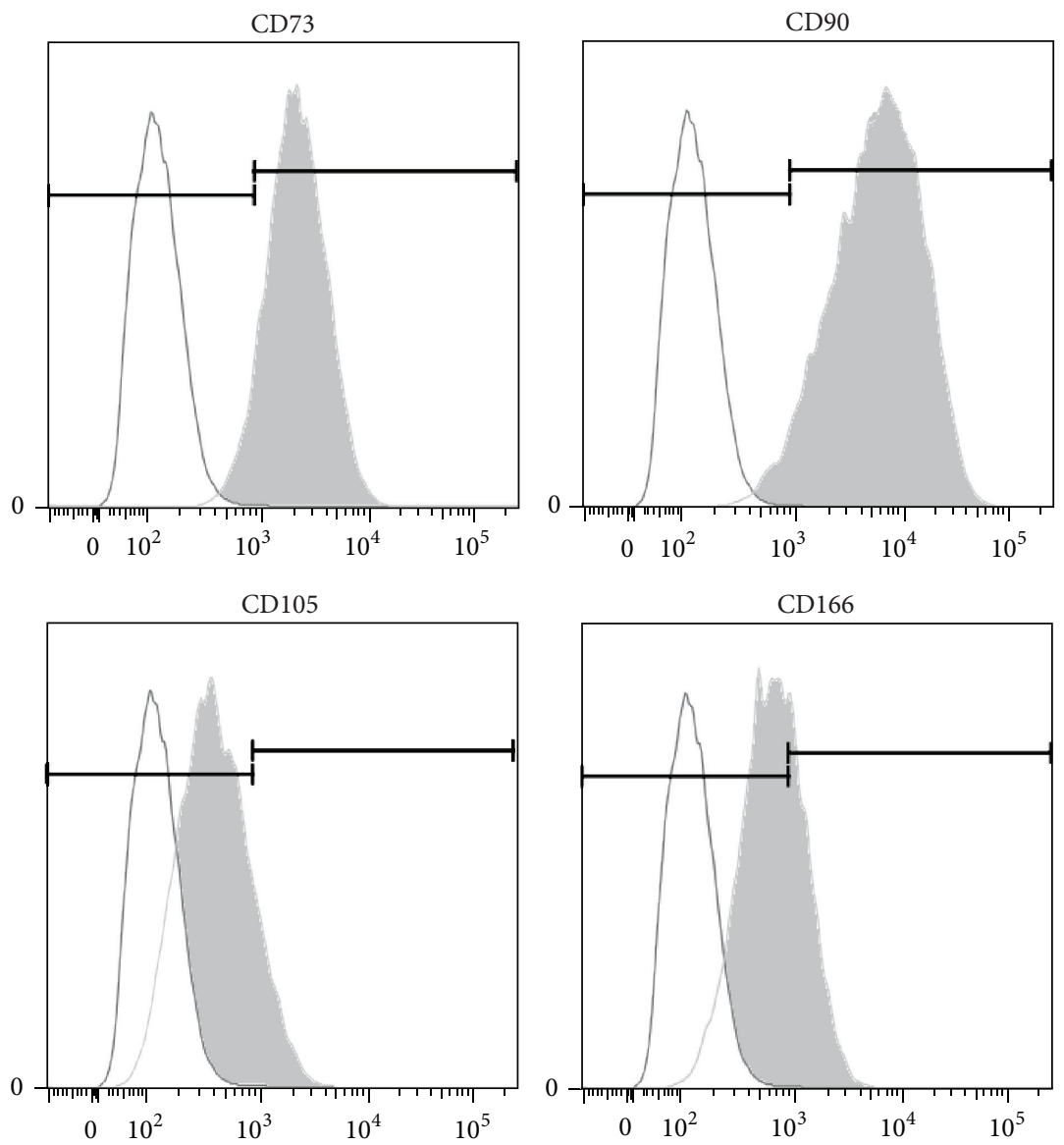

(b)

Figure 1: (a) A microscope image of cultured hDPSCs isolated from human primary teeth with no induction. (b) Positive expression of several cell surface antigens for mesenchymal stem cells, including CD73, CD90, CD105, and CD166, in hDPSCs. Similar cell surface antigen expression pattern was obtained with hDPSCs isolated from different donors (data not shown).

ANOVA with Tukey's post hoc test for multiple comparisons. In all analyses, $P<0.05$ indicated statistically significant differences between values.

\section{Results}

3.1. Mesenchymal Stem Cell Markers Expressed in Cultured hDPSCs. Human DPSCs from postnatal human primary teeth were used to explore the functional role of MSX1. These cells exhibited a fibroblastic shape (Figure 1(a)) and showed expression of mesenchymal stem cell surface markers CD73 (>90\%), CD90 (>90\%), CD105 (>10\%), and CD166 (>30\%) (Figure 1(b)) as expected from previous studies [24].

3.2. MSX1 Knockdown Abolishes Osteogenic Differentiation of hDPSCs. Human DPSCs were transfected with two 

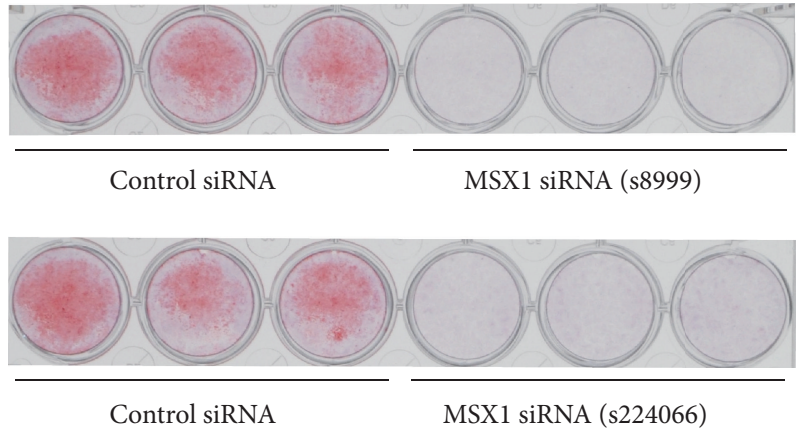

(a)

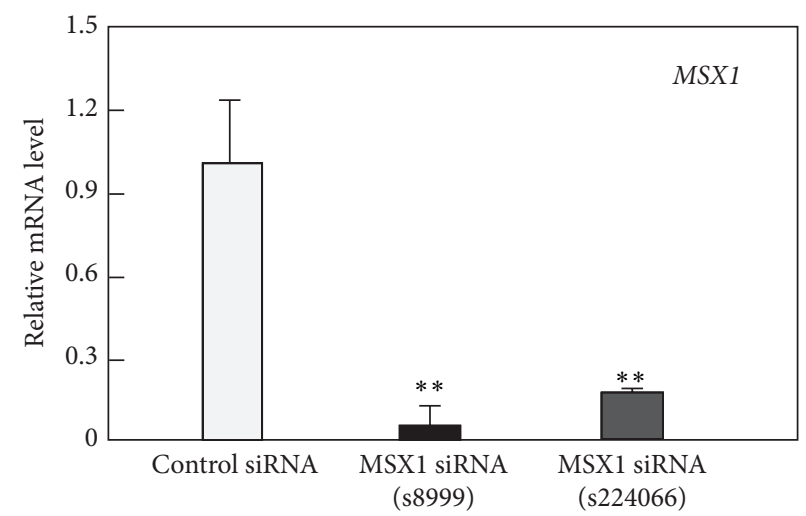

(b)

FIGURE 2: Effects of MSX1 knockdown on calcification of hDPSCs. hDPSCs were transfected with either MSX1 siRNA (s8999 or s224066) or control siRNA and incubated for 2 days in growth medium before the cultures became confluent. Thereafter, the cultures were exposed to osteogenesis-induction medium. (a) The calcified matrix was stained with alizarin red on day 14. (b) MSX1 mRNA level was quantified by RT-qPCR at $48 \mathrm{~h}$ after transfection. Values are averages \pm SD for three cultures. ${ }^{* *} P<0.01$.

different siRNAs for MSX1 or control siRNA and then exposed to osteogenesis-induction medium. Both siRNA oligonucleotides targeting MSX1 (s8999 and s224066) abolished MSX1 mRNA expression at $48 \mathrm{~h}$ and subsequent matrix calcification on day 14 (Figure 2). We selected MSX1 siRNA (s8999) for subsequent studies.

Next, we examined the effect of MSX1 knockdown on alkaline phosphatase activity and the expression of osteoblast-related genes in hDPSCs after the onset of osteogenesis (Figure 3). In hDPSCs transfected with control siRNA, alkaline phosphatase activity and RUNX2, BMP2, osterix (OSX), osteocalcin (OCN; also known as BGLAP), and alkaline phosphatase liver type $(A L P L)$ mRNA levels increased on days 4-12 after the onset of differentiation. MSX1 mRNA levels also increased on days 4-12. However, MSX1 knockdown abolished the induction of alkaline phosphatase activity (Figure 3(a)) and the increases in ALPL, RUNX2, BMP2, $O C N$, and MSX1 mRNA levels, although it further increased OSX mRNA levels (Figure 3(b)). It should be noted that the incubation with MSX1 siRNA abolished MSX1 expression at least until day 12 after the onset of osteogenic differentiation.

Next, we examined whether MSX1 knockdown might influence the expression of other master genes including a master regulator of chondrogenesis SOX9 and a master regulator of adipogenesis PPAR $\gamma$ (Figure 4). In control hDPSCs, no significant changes in the expressions of SOX9 and PPAR $\gamma$ were observed after the exposure to osteogenesis-induction medium. Under these conditions, MSX1 knockdown increased the expression of PPAR $\gamma$ on days $4-8$, although it had little effect on the expression level of SOX9.

3.3. MSX1 Knockdown Downregulated and Upregulated a Variety of Genes. To characterize the effects of MSX1 knockdown on osteogenic differentiation, we performed DNA microarray analyses on day 4 after exposure to osteogenesisinduction medium. MSX1 knockdown decreased and increased mRNA levels of 2923 and 3480 genes, respectively, which were selected with cut-off values of $>1.5$-fold change and $t$-test $P<0.05$. Tables 2 and 3 show lists of downregulated and upregulated genes in MSX1-knockdown hDPSCs, respectively.

To understand MSX1 actions in hDPSCs differentiating into osteoblasts, we performed a gene-set approach using the 2923 downregulated and 3480 upregulated genes. The WikiPathways analysis showed that the MSX1 knockdown downregulated various genes involved in focal adhesion, endochondral ossification, integrin-mediated cell adhesion, matrix metalloproteinases, calcium regulation, and insulin signaling (Table 4), whereas it upregulated genes involved in sterol regulatory element-binding protein (SREBP) signaling, cholesterol biosynthesis, adipogenesis, and fatty acid biosynthesis (Table 5). These findings revealed that MSX1 regulates various cellular processes in hDPSCs differentiating into osteoblasts.

3.4. MSX1 Knockdown Upregulates Cholesterol SynthesisRelated Genes. In MSX1-knockdown hDPSCs, "SREBP signaling" and "cholesterol biosynthesis" were the top 1st and 3rd upregulated gene sets, respectively (Table 5). The SREBP2 master transcriptional factor regulates the expression of all genes encoding enzymes in cholesterol synthesis pathway [25]. Because cholesterol synthesis is closely linked with osteoblast differentiation [17], we examined the effect of MSX1 knockdown on the expression of these genes. DNA microarray analyses showed that all cholesterol synthesisrelated genes, including $S R E B P 2$, were significantly upregulated by MSX1 knockdown on day 4 (Figure 5(a)). Quantitative RT-PCR analyses confirmed that MSX1 knockdown increased SREBP2, 3-hydroxy-3-methylglutaryl-CoA synthase 1 (HMGCS1), HMG-CoA reductase (HMGCR), farnesyl diphosphate synthase (FDPS), Cytochrome P450 Family 51 Subfamily A Polypeptide 1 (CYP51A1), and 7-dehydrocholesterol reductase (DHCR7) mRNA levels (Figure 5(b)). 


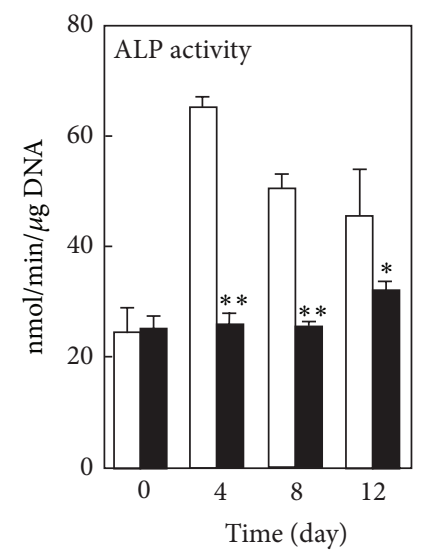

$\square$ Control siRNA $\rceil$ *

[ MSX1 siRNA ]**

(a)
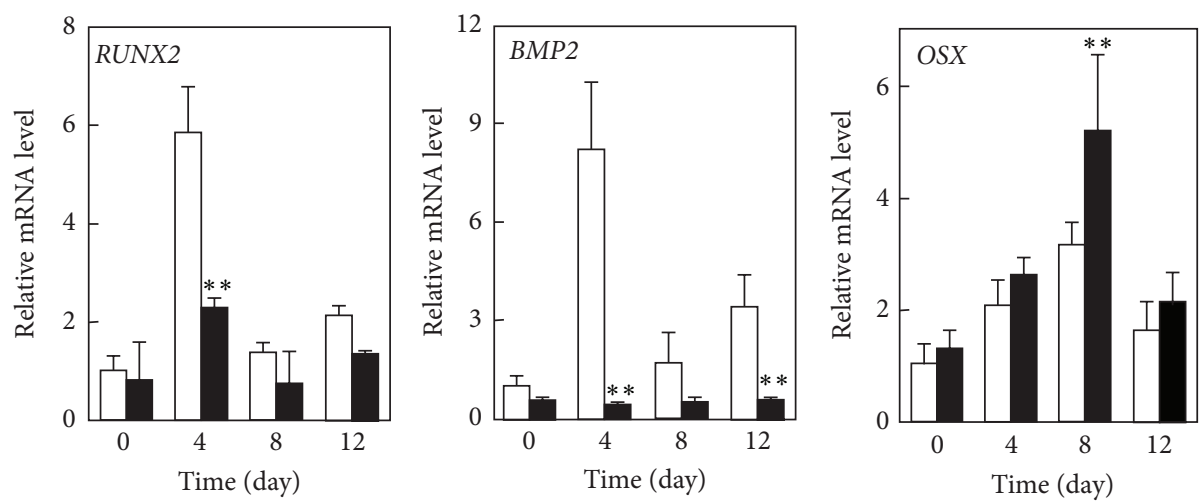

$\left.\begin{array}{l}\square \text { Control siRNA } \\ \text { MSX1 siRNA }\end{array}\right] * *$

$\left.\begin{array}{l}\text { Control siRNA } \\ \text { MSX1 siRNA }\end{array}\right] * *$

Control siRNA $]_{*}$

- MSX1 siRNA $]^{* *}$
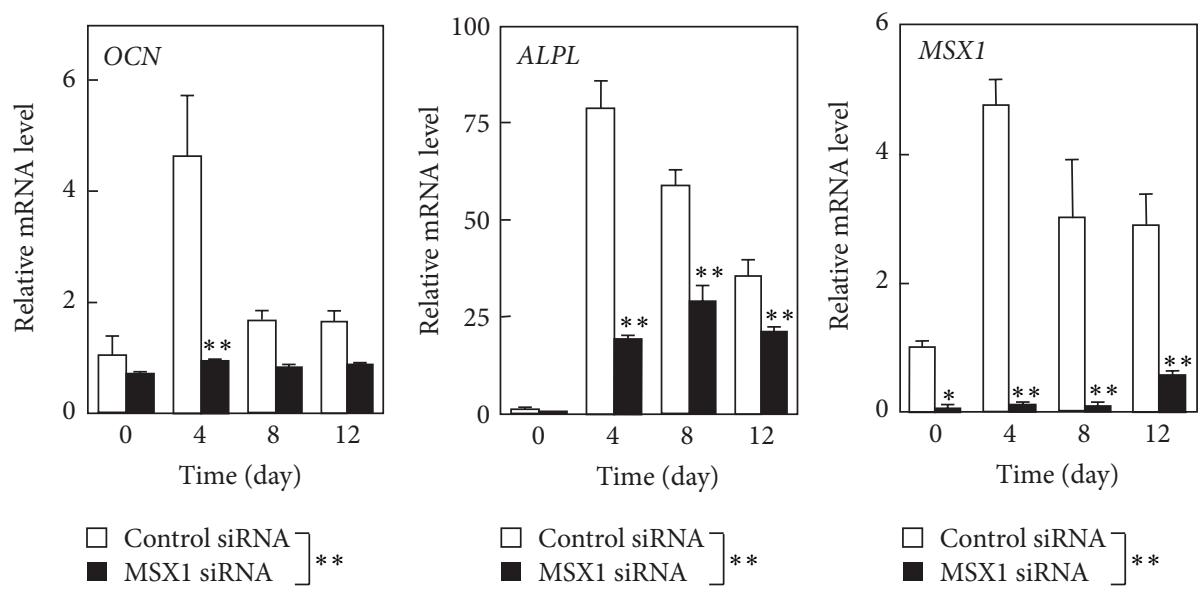

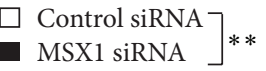

(b)

FIGURE 3: Effects of MSX1 knockdown on the expression of osteogenic markers in hDPSCs. (a) Alkaline phosphatase activity in cultures was determined on days 0-12 after exposure to osteogenesis-induction medium. (b) The mRNA levels of osteoblast-related genes, including RUNX2, BMP2, OSX, OCN, and ALPL, along with the MSX1 mRNA level were quantified by RT-qPCR on the indicated days. Values are averages \pm SD for three cultures. ${ }^{*} P<0.05 ;{ }^{* *} P<0.01$. 
TABLE 2: The list of downregulated genes in MSX1-knockdown cells (top 50).

\begin{tabular}{|c|c|c|c|}
\hline Gene symbol & Gene name & Gene ID & Fold change \\
\hline C11orf96 & Chromosome 11 open reading frame 96 & NM_001145033 & 92.53 \\
\hline JAM2 & Junctional adhesion molecule 2 & NM_021219 & 70.90 \\
\hline MLC1 & Megalencephalic leukoencephalopathy with subcortical cysts 1 & NM_015166 & 65.56 \\
\hline NPPC & Natriuretic peptide C & NM_024409 & 54.40 \\
\hline CPXM1 & Carboxypeptidase X (M14 family), member 1 & NM_019609 & 51.08 \\
\hline EFCC1 & EF-hand and coiled-coil domain containing 1 & NM_024768 & 44.56 \\
\hline SFRP4 & Secreted frizzled-related protein 4 & NM_003014 & 43.96 \\
\hline JAM2 & Junctional adhesion molecule 2 & NM_021219 & 38.31 \\
\hline LOC200772 & Uncharacterized LOC200772 & NR_033841 & 34.41 \\
\hline S100A8 & S100 calcium binding protein A8 & NM_002964 & 31.72 \\
\hline JAM2 & Junctional adhesion molecule 2 & NM_001270408 & 31.42 \\
\hline CPXM1 & Carboxypeptidase X (M14 family), member 1 & NM_019609 & 29.44 \\
\hline SFRP4 & Secreted frizzled-related protein 4 & NM_003014 & 28.11 \\
\hline KIT & v-kit Hardy-Zuckerman 4 feline sarcoma viral oncogene homolog & NM_000222 & 27.48 \\
\hline CLCA2 & Chloride channel accessory 2 & NM_006536 & 24.88 \\
\hline LINC00473 & Long intergenic nonprotein coding RNA 473 & NR_026860 & 24.34 \\
\hline ST8SIA4 & ST8 alpha-N-acetyl-neuraminide alpha-2,8-sialyltransferase 4 & NM_005668 & 24.16 \\
\hline TEX29 & Testis expressed 29 & NM_152324 & 23.52 \\
\hline PTGDR2 & Prostaglandin D2 receptor 2 & NM_004778 & 23.31 \\
\hline CXCL14 & Chemokine (C-X-C motif) ligand 14 & NM_004887 & 22.63 \\
\hline HS6ST2 & Heparan sulfate 6-O-sulfotransferase 2 & NM_001077188 & 22.61 \\
\hline CHST15 & $\begin{array}{l}\text { Carbohydrate (N-acetylgalactosamine 4-sulfate 6-O) Sulfotransferase } \\
15\end{array}$ & NM_015892 & 21.84 \\
\hline PIANP & PILR alpha associated neural protein & NM_153685 & 21.67 \\
\hline SNAP25 & Synaptosomal-associated protein, $25 \mathrm{kDa}$ & NM_003081 & 21.55 \\
\hline CBLN2 & Cerebellin 2 precursor & NM_182511 & 21.30 \\
\hline FRAS1 & Fraser syndrome 1 & NM_025074 & 21.24 \\
\hline SECTM1 & Secreted and transmembrane 1 & NM_003004 & 20.76 \\
\hline NFE2 & Nuclear factor, erythroid 2 & NM_006163 & 20.46 \\
\hline GABBR2 & Gamma-aminobutyric acid (GABA) B receptor, 2 & NM_005458 & 19.22 \\
\hline MSX1 & Msh homeobox 1 & NM_002448 & 19.08 \\
\hline SCARA5 & Scavenger receptor class A, member 5 (putative) & NM_173833 & 18.79 \\
\hline PRSS35 & Protease, serine, 35 & NM_153362 & 18.60 \\
\hline WNT2B & Wingless-type MMTV integration site family, member 2B & NM_004185 & 17.95 \\
\hline BMP2 & Bone morphogenetic protein-2 & NM_001200 & 17.63 \\
\hline NDRG4 & NDRG family member 4 & NM_022910 & 17.39 \\
\hline CRTAM & Cytotoxic and regulatory $\mathrm{T}$ cell molecule & NM_019604 & 16.80 \\
\hline RASL12 & RAS-like, family 12 & NM_016563 & 15.91 \\
\hline THBD & Thrombomodulin & NM_000361 & 15.26 \\
\hline CHST1 & Carbohydrate (keratan sulfate Gal-6) sulfotransferase 1 & NM_003654 & 14.90 \\
\hline DIO3OS & DIO3 opposite strand/antisense RNA (head to head) & NR_002770 & 14.81 \\
\hline CCR1 & Chemokine (C-C motif) receptor 1 & NM_001295 & 14.81 \\
\hline TMEM35 & Transmembrane protein 35 & NM_021637 & 14.79 \\
\hline HAS1 & Hyaluronan synthase 1 & NM_001523 & 14.68 \\
\hline SCN1B & Sodium channel, voltage-gated, type I, beta subunit & NM_199037 & 14.34 \\
\hline ADAMTS17 & ADAM metallopeptidase with thrombospondin type 1 motif, 17 & NM_139057 & 14.12 \\
\hline GALNT15 & $\begin{array}{l}\text { UDP-N-acetyl-alpha-D-galactosamine:polypeptide } \\
\mathrm{N} \text {-acetylgalactosaminyltransferase } 15\end{array}$ & NM_054110 & 13.86 \\
\hline $\mathrm{RHOH}$ & Ras homolog family member $\mathrm{H}$ & NM_004310 & 13.80 \\
\hline
\end{tabular}


TABLe 2: Continued.

\begin{tabular}{llcc}
\hline Gene symbol & Gene name & Gene ID & Fold change \\
\hline GPR68 & G protein-coupled receptor 68 & NM_003485 & 13.63 \\
TAC3 & Tachykinin 3 & NM_013251 & 13.37 \\
MIR1247 & MicroRNA 1247 & AF469204 & 13.13 \\
\hline
\end{tabular}
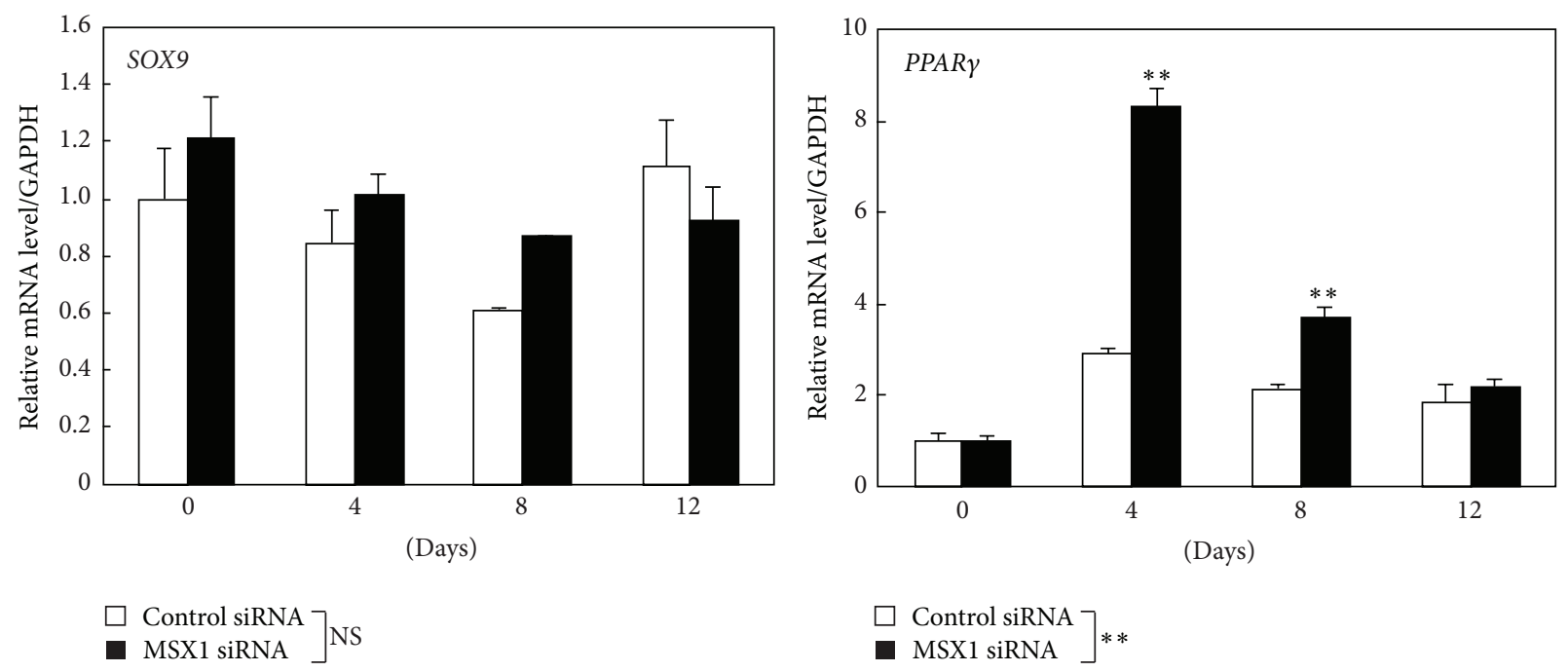

FIGURE 4: Effects of MSX1 knockdown on the expression of the master genes of chondrogenesis and adipogenesis in hDPSCs. The mRNA levels of SOX9 and PPAR $\gamma$ in hDPSCs transfected with MSX1 siRNA or control siRNA were quantified by RT-qPCR on the indicated days. Values are averages \pm SD for 3 cultures. ${ }^{* *} P<0.01$; NS: not significant.

\section{Discussion}

Previous studies showed that mouse MSX1 was implicated in craniofacial bone development $[1,2,4]$. In mouse embryos, MSX1 suppresses precocious differentiation and calcification in dental mesenchymal cells and maintains these cells in a proliferative state to ensure subsequent craniofacial and tooth development $[6,26]$. High levels of osteoblast number, cell proliferation, and apoptosis in MSX1 transgenic mice suggest that MSX1 modulates mouse craniofacial bone modeling [4]. However, the role of MSX1 in human cells remains poorly understood. In the present study, we demonstrated that MSX1 plays an essential role in osteogenic differentiation of hDPSCs.

In human DPSC cultures, MSX1 knockdown resulted in suppressed expression of RUNX2, ALPL, BMP2, and OCN. These results demonstrate that MSX1 modulated the major signaling/transcriptional pathways regulating hard tissue differentiation to enhance osteogenic potential of hDPSCs. However, MSX1 knockdown unexpectedly increased the mRNA level of OSX, another transcriptional factor involved in osteoblast maturation. This indicates MSX1 does not activate the entire osteogenesis program, perhaps because MSX1 cooperates with other transcription factors to fully control osteogenesis. MSX1 knockdown enhanced PPAR $\gamma$ expression under the osteogenesis-induced condition, suggesting that MSX1 negatively regulates adipogenic differentiation. MSX1 may direct hDPSCs into the osteoblast lineage by preventing them from differentiating into the adipogenic lineage. MSX1 knockdown also resulted in downregulation of various genes involved in focal adhesion, integrin-mediated cell adhesion, matrix metalloproteinases, calcium regulation, insulin signaling, and other processes. The extensive effect of MSX1 knockdown on the entire gene expression profile emphasizes a crucial role of MSX1 in hDPSCs undergoing differentiation into osteoblasts.

Bidirectional transcription of the $M s x 1$ gene has been previously reported [27-29]. In embryonic and newborn mice, sense and antisense Msxl transcripts are differently expressed during development. In 705IC5 mouse odontoblasts, overexpression of $M s x 1$ antisense RNA decreased the expression of Msxl sense transcript, whereas overexpression of $M s x 1$ sense RNA increased $M s x 1$ antisense transcript. Thus, expression of mouse $M s x 1$ is controlled by the balance of the two transcripts. In our experiments, however, MSX1 antisense transcript was not detected during osteogenic differentiation of hDPSCs irrespective of siRNA knockdown of MSX1 (data not shown). The presence of MSX1 antisense transcript in humans has so far been reported only in the embryo. Therefore, $M s x 1$ antisense RNA does not seem to be involved in the MSX1 expression in hDPSCs. Under these conditions, the expression of $M s x 1$ sense transcripts was markedly depressed in hDPSCs after treatment with MSX1 siRNA, indicating that the knockdown experiments worked appropriately regardless of the presence or absence of the Msxl antisense transcript.

MSX2, a paralog of MSX1, has been shown to enhance osteogenic differentiation of various mesenchymal cells, 
TABLE 3: The list of upregulated genes in MSX1-knockdown cells (top 50).

\begin{tabular}{|c|c|c|c|}
\hline Gene symbol & Gene name & Gene ID & Fold change \\
\hline MLXIPL & MLX interacting protein-like & NM_032951 & 74.63 \\
\hline CRLF1 & Cytokine receptor-like factor 1 & NM_004750 & 73.44 \\
\hline ATP1A2 & ATPase, $\mathrm{Na}+/ \mathrm{K}+$ transporting, alpha 2 polypeptide & NM_000702 & 69.84 \\
\hline MAP2 & Microtubule-associated protein 2 & NM_002374 & 62.64 \\
\hline PRODH & Proline dehydrogenase (oxidase) 1 & NM_016335 & 50.32 \\
\hline LONRF3 & LON peptidase $\mathrm{N}$-terminal domain and ring finger 3 & NM_001031855 & 30.84 \\
\hline ERICH2 & Glutamate-rich 2 & XM_001714892 & 29.67 \\
\hline $\mathrm{DMD}$ & Dystrophin & NM_004010 & 27.91 \\
\hline SAA1 & Serum amyloid A1 & NM_000331 & 26.65 \\
\hline CLDN20 & Claudin 20 & NM_001001346 & 24.19 \\
\hline DMD & Dystrophin & NM_004021 & 21.88 \\
\hline PLIN4 & Perilipin 4 & NM_001080400 & 21.64 \\
\hline RLBP1 & Retinaldehyde binding protein 1 & NM_000326 & 21.22 \\
\hline SAA2 & Serum amyloid A2 & NM_030754 & 20.98 \\
\hline PARD6B & Par-6 family cell polarity regulator beta & NM_032521 & 19.56 \\
\hline ANO3 & Anoctamin 3 & NM_031418 & 17.58 \\
\hline KCNJ16 & Potassium inwardly rectifying channel, subfamily J, member 16 & NM_170741 & 17.41 \\
\hline LOC284561 & Uncharacterized LOC284561 & XR_110828 & 16.52 \\
\hline USP53 & Ubiquitin specific peptidase 53 & NM_019050 & 16.47 \\
\hline CLGN & Calmegin & NM_004362 & 16.35 \\
\hline USP53 & Ubiquitin specific peptidase 53 & NM_019050 & 16.15 \\
\hline PLCE1-AS1 & PLCE1 antisense RNA 1 & NR_033969 & 15.15 \\
\hline KLHDC7B & Kelch domain containing 7B & NM_138433 & 14.44 \\
\hline PSG9 & Pregnancy specific beta-1-glycoprotein 9 & NM_002784 & 14.23 \\
\hline ERICH2 & Glutamate-rich 2 & XM_001714892 & 13.66 \\
\hline ANKRD1 & Ankyrin repeat domain 1 (cardiac muscle) & NM_014391 & 13.58 \\
\hline PDE6A & Phosphodiesterase 6A, cGMP-specific, rod, alpha & NM_000440 & 13.23 \\
\hline COL4A4 & Collagen, type IV, alpha 4 & NM_000092 & 12.98 \\
\hline PLAC8 & Placenta-specific 8 & NM_016619 & 12.85 \\
\hline BEST2 & Bestrophin 2 & NM_017682 & 12.72 \\
\hline IP6K3 & Inositol hexakisphosphate kinase 3 & NM_054111 & 12.22 \\
\hline DNAH2 & Dynein, axonemal, heavy chain 2 & NM_020877 & 12.07 \\
\hline INHBB & Inhibin, beta B & NM_002193 & 11.63 \\
\hline LOC100506544 & Uncharacterized LOC100506544 & AK057177 & 10.9 \\
\hline TMEM125 & Transmembrane protein 125 & NM_144626 & 10.57 \\
\hline ORM2 & Orosomucoid 2 & NM_000608 & 10.51 \\
\hline PCSK9 & Proprotein convertase subtilisin/kexin type 9 & NM_174936 & 10.17 \\
\hline CD200 & CD200 molecule & NM_001004196 & 9.99 \\
\hline MFSD2A & Major facilitator superfamily domain containing $2 \mathrm{~A}$ & NM_001136493 & 9.85 \\
\hline IL8 & Interleukin 8 & NM_000584 & 9.80 \\
\hline FMO6P & Flavin containing monooxygenase 6 pseudogene & NR_002601 & 9.60 \\
\hline C6 & Complement component 6 & NM_000065 & 9.54 \\
\hline DSCR8 & Down syndrome critical region gene 8 & NR_026838 & 9.23 \\
\hline LOC648149 & Uncharacterized LOC648149 & AK123349 & 9.19 \\
\hline GPR18 & G protein-coupled receptor 18 & NM_005292 & 9.14 \\
\hline ORM1 & Orosomucoid 1 & NM_000607 & 9.04 \\
\hline GATA3 & GATA binding protein 3 & NM_001002295 & 9.03 \\
\hline KCNB1 & Potassium voltage-gated channel, Shab-related subfamily, member 1 & NM_004975 & 8.97 \\
\hline
\end{tabular}


TABLE 3: Continued.

\begin{tabular}{llcc}
\hline Gene symbol & Gene name & Gene ID & Fold change \\
\hline OCA2 & Oculocutaneous albinism II & NM_000275 & 8.90 \\
PDZRN4 & PDZ domain containing ring finger 4 & NM_013377 & 8.86 \\
\hline
\end{tabular}

TABLE 4: The WikiPathways analysis selected gene sets significantly downregulated in MSX1-knockdown cells.

\begin{tabular}{|c|c|c|c|}
\hline Ranking & Pathway & $P$ value & Gene counts (gene number of pathway) \\
\hline 1 & Focal adhesion & $4.20 E-09$ & $36(188)$ \\
\hline 2 & IL-4 signaling pathway & $2.55 E-05$ & $13(55)$ \\
\hline 3 & Endochondral ossification & $6.27 E-05$ & $14(64)$ \\
\hline 4 & Muscle cell tarbase & $7.95 E-05$ & $41(336)$ \\
\hline 5 & Integrin-mediated cell adhesion & $8.22 E-05$ & $18(99)$ \\
\hline 6 & Matrix metalloproteinases & $9.72 E-05$ & $9(31)$ \\
\hline 7 & Regulation of toll-like receptor signaling pathway & $1.12 E-04$ & $22(150)$ \\
\hline 8 & Calcium regulation in the cardiac cell & $1.30 E-04$ & $23(149)$ \\
\hline 9 & MicroRNAs in cardiomyocyte hypertrophy & $3.35 E-04$ & $15(105)$ \\
\hline 10 & Insulin signaling & $4.14 E-04$ & $23(161)$ \\
\hline
\end{tabular}

TABLE 5: The WikiPathways analysis selected gene sets significantly upregulated in MSX1 knockdown cells.

\begin{tabular}{lccc}
\hline Ranking & Pathway & $P$ value & Gene counts (gene number of pathway) \\
\hline 1 & SREBP signalling & $<1.00 E-44$ & $24(50)$ \\
2 & Lymphocyte tarbase & $<1.00 E-44$ & $78(420)$ \\
3 & Cholesterol biosynthesis & $1.86 E-17$ & $16(17)$ \\
4 & Muscle cell tarbase & $1.78 E-10$ & $65(336)$ \\
5 & Epithelium tarbase & $9.47 E-10$ & $52(278)$ \\
6 & Folate metabolism & $1.47 E-09$ & $22(68)$ \\
7 & Adipogenesis & $3.19 E-08$ & $30(131)$ \\
8 & Leukocyte tarbase & $1.47 E-07$ & $28(128)$ \\
9 & Fatty acid biosynthesis & $1.73 E-06$ & $10(22)$ \\
\hline
\end{tabular}

including C3H10T1/2 cells and aorta myofibroblasts [30-32]. MSX1 and MSX2 activate aortic adventitial osteoprogenitors via overlapping yet distinct mechanisms [33]. MSX2, unlike MSX1, enhances OSX expression without an increase in RUNX2 expression in aortic myofibroblasts [30], suggesting distinct actions of MSX1 and MSX2 in osteoblast differentiation.

The molecular mechanism by which MSX1 activates the differentiation program remains unclear. MSX1 regulates transcriptional activity of target genes either by directly binding to the specific DNA MSX1-binding motif (C/GTAATTG) or through interactions with other transcriptional regulators. Interestingly, MSX1 binds to various transcriptional regulators, including Sp1, Sp3, Dlx3, Dlx5, PAX3, PAX9, BarH-like homeobox 1/BARX1, and PIAS1 [34-37]. Depending on the partner in the complex, MSX1 activates or represses transcription in the MSX1-interacting network of transcription factors [26, 38]. Moreover, MSX1 modifies chromatin structure near target genes by histone methylation $[35,39]$. The interactions of MSX1 with various transcriptional regulators may account for the extensive changes in the expression levels of many genes ( 6400) by MSX1 knockdown.

Statins, drugs for hyperlipidemia, enhance osteogenic differentiation of various mesenchymal cells, including osteoblast precursor cells, mesenchymal stem cells, and DPSCs, by inhibiting the synthesis of farnesyl pyrophosphate, decreasing cellular cholesterol, and activating the Ras-PI3KAkt/MAPK signaling pathway, thereby increasing the expression of BMP2 and RUNX2 [17], although the underlying mechanisms are still controversial. Statins also suppress osteoclast function and enhance mandibular bone formation in vivo [40]. Interestingly, a previous study showed that simvastatin induces odontoblast differentiation of hDPSCs in vitro and in vivo [22]. However, no studies have shown the involvement of transcription factor(s) in the control of cholesterol synthesis during osteoblast differentiation. Here we found for the first time that MSX1 suppresses the entire cholesterol synthesis pathway in osteoblast differentiating hDPSCs by repressing SREBP2 and other related genes. This suppression of cholesterol synthesis may facilitate osteoblast 


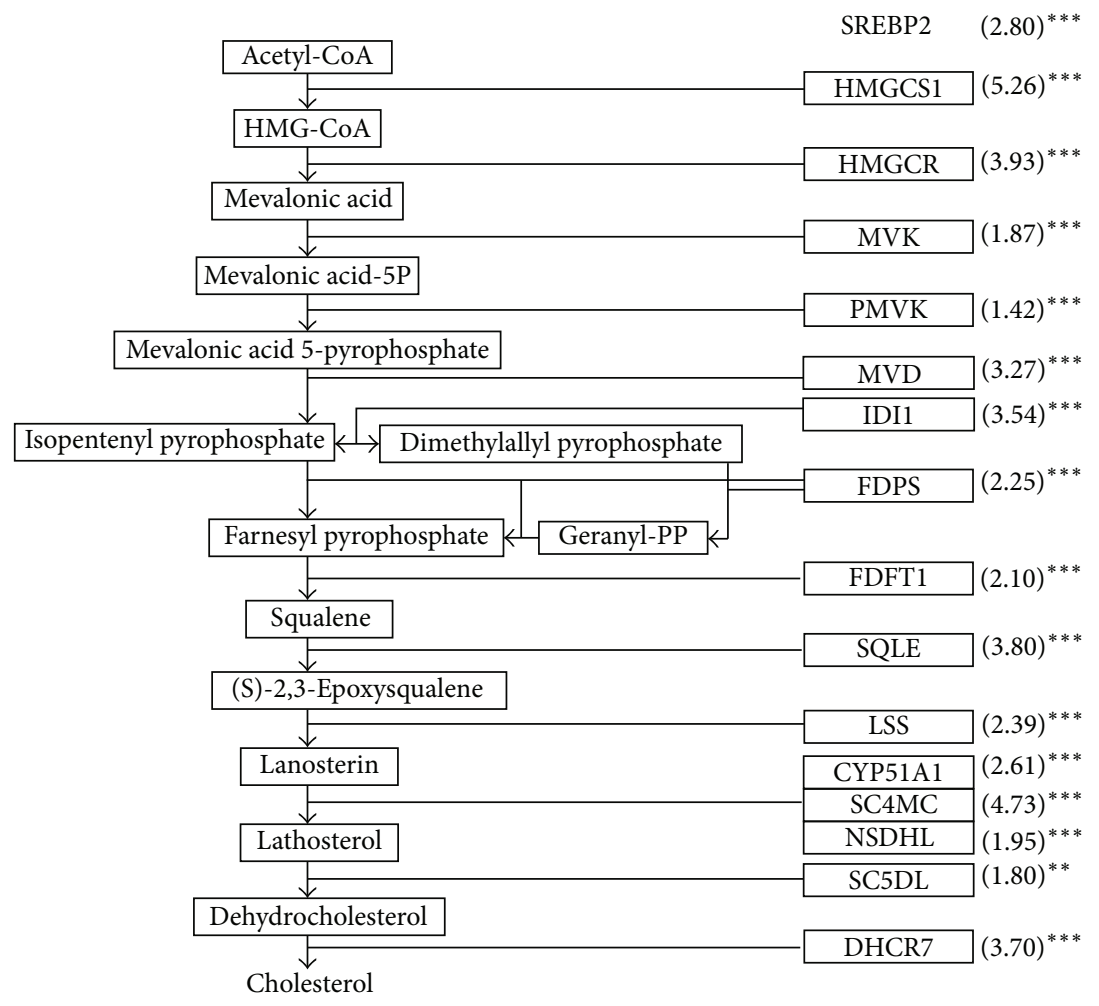

(a)

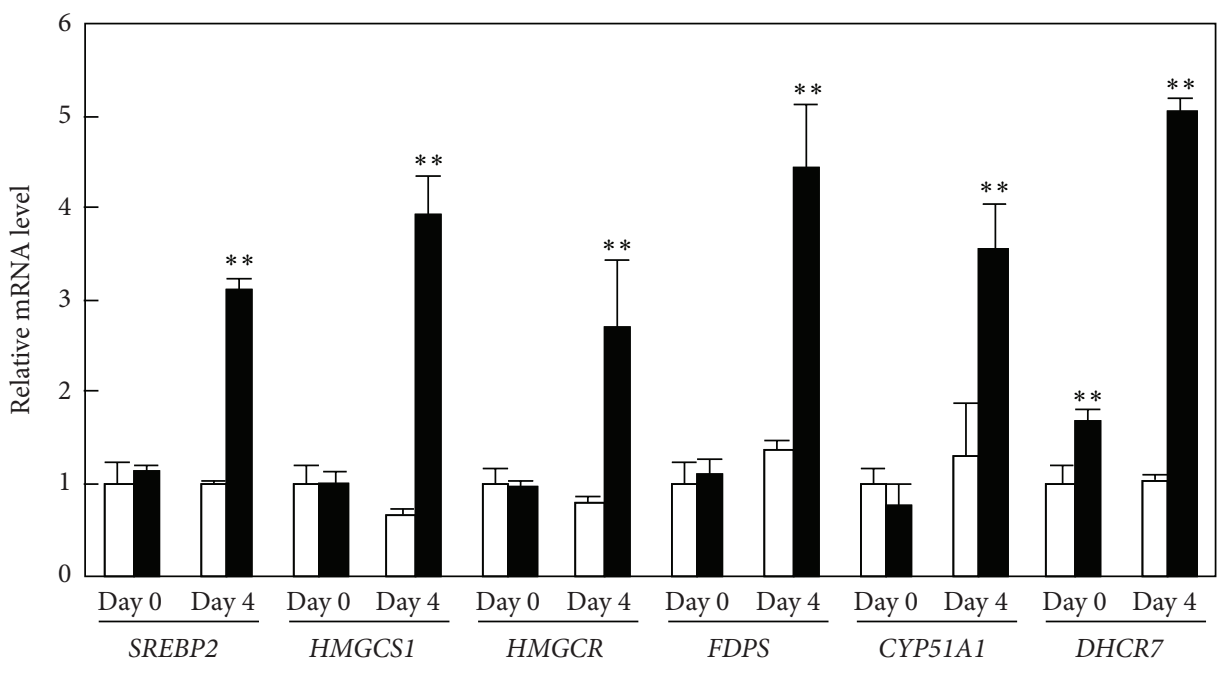

$\square$ Control siRNA

MSX1 siRNA

(b)

FIGURE 5: Effects of MSX1 knockdown on the expression of cholesterol synthesis-related genes, which are direct targets for SREBP2, in hDPSCs. (a) The cholesterol biosynthesis pathway is shown and the bold frames indicate target genes for the master transcriptional factor SREBP2. Microarray analysis indicates all genes involved in cholesterol synthesis are upregulated by MSX1 knockdown. The numbers in brackets represent the fold changes in gene expression in MSX1-knockdown cells as compared with the control cells. (b) The mRNA levels of genes relevant to cholesterol synthesis, including SREBP2, HMGCS1, HMGCR, FDPS, CYP51A1, and DHCR7, were quantified by RT-qPCR analysis. Values are averages $\pm \mathrm{SD}$ for three cultures. ${ }^{* *} \mathrm{P}<0.01$; ${ }^{* * *} \mathrm{P}<0.001$. 
differentiation. It is also interesting to note that various mutations in the cholesterol synthesis pathway, including 7dehydrocholesterol reductase (DHCR7), cause craniofacial anomalies including cleft palate, suggesting the role of cholesterol synthesis in craniofacial development [41].

In conclusion, here we revealed for the first time that MSX1 is indispensable for osteoblast-like differentiation and calcification in hDPSCs derived from deciduous teeth. Furthermore, MSX1 was found to modulate a wide variety of genes, including cholesterol synthesis-related genes, during osteogenic differentiation of hDPSCs. We have not examined the effects of MSX1 siRNA in definitive teeth, although MSX1 may also function as a positive regulator of osteogenesis in definitive teeth as MSX1 mRNA levels are high in both definitive and deciduous teeth [15]. Our findings will provide new insights into the role of MSX1 in development and repair of teeth and may be useful in DPSC-based regenerative therapy.

\section{Competing Interests}

The authors declare that there is no conflict of interests regarding the publication of this paper.

\section{Acknowledgments}

This work was supported by Grant-in-Aid for Challenging Exploratory Research (no. 24659876), Scientific Research (C; no. 25462889), and Grant-in-Aid for Young Scientists (B; no. 15K20592) from the Ministry of Education, Culture, Sports, Science and Technology of Japan to Yukio Kato, Katsumi Fujimoto, and Noriko Goto, respectively. The authors would like to thank Dr. Eiso Hiyama at the Natural Science Center for Basic Research and Development and Hiroshima University for use of the equipment.

\section{References}

[1] I. Satokata and R. Maas, "Msxl deficient mice exhibit cleft palate and abnormalities of craniofacial and tooth development," Nature Genetics, vol. 6, no. 4, pp. 348-356, 1994.

[2] R. Maas, Y. P. Chen, M. Bei, I. Woo, and I. Satokata, "The role of Msx genes in mammalian development," Annals of the New York Academy of Sciences, vol. 785, pp. 171-181, 1996.

[3] S. M. Orestes-Cardoso, J. R. Nefussi, D. Hotton et al., "Postnatal Msxl expression pattern in craniofacial, axial, and appendicular skeleton of transgenic mice from the first week until the second year," Developmental Dynamics, vol. 221, no. 1, pp. 1-13, 2001.

[4] A. Nassif, I. Senussi, F. Meary et al., "Msxl role in craniofacial bone morphogenesis," Bone, vol. 66, pp. 96-104, 2014.

[5] A. Mackenzie, G. L. Leeming, A. K. Jowett, M. W. J. Ferguson, and P. T. Sharpe, "The homeobox gene Hox 7.1 has specific regional and temporal expression patterns during early murine craniofacial embryogenesis, especially tooth development in vivo and in vitro," Development, vol. 111, no. 2, pp. 269-285, 1991.

[6] J. Han, Y. Ito, J. Y. Yeo, H. M. Sucov, R. Maas, and Y. Chai, "Cranial neural crest-derived mesenchymal proliferation is regulated by Msxl-mediated p19INK4d expression during odontogenesis," Developmental Biology, vol. 261, no. 1, pp. 183-196, 2003.
[7] Y. Chen, M. Bei, I. Woo, I. Satokata, and R. Maas, "Msxl controls inductive signaling in mammalian tooth morphogenesis," Development, vol. 122, no. 10, pp. 3035-3044, 1996.

[8] M. Vieux-Rochas, K. Bouhali, S. Mantero et al., "BMP-mediated functional cooperation between Dlx5;Dlx6 and Msx1;Msx2 during mammalian limb development," PLoS ONE, vol. 8, no. 1, Article ID e51700, 2013.

[9] H. Vastardis, N. Karimbux, S. W. Guthua, J. G. Seidman, and C. E. Seidman, "A human MSX1 homeodomain missense mutation causes selective tooth agenesis," Nature Genetics, vol. 13, no. 4, pp. 417-421, 1996.

[10] S. Yamaguchi, J. Machida, M. Kamamoto et al., "Characterization of novel MSX1 mutations identified in Japanese patients with nonsyndromic tooth agenesis," PLoS ONE, vol. 9, no. 8, article e102944, 2014.

[11] R. Nakatsuka, T. Nozaki, Y. Uemura et al., “5-Aza-2'-deoxycytidine treatment induces skeletal myogenic differentiation of mouse dental pulp stem cells," Archives of Oral Biology, vol. 55, no. 5, pp. 350-357, 2010.

[12] K. Ranganathan and V. Lakshminarayanan, "Stem cells of the dental pulp," Indian Journal of Dental Research, vol. 23, no. 4, article 558, 2012.

[13] K. Iohara, K. Imabayashi, R. Ishizaka et al., "Complete pulp regeneration after pulpectomy by transplantation of CD105+ stem cells with stromal cell-derived factor-1," Tissue Engineering Part A, vol. 17, no. 15-16, pp. 1911-1920, 2011.

[14] K. Sakai, A. Yamamoto, K. Matsubara et al., "Human dental pulp-derived stem cells promote locomotor recovery after complete transection of the rat spinal cord by multiple neuroregenerative mechanisms," Journal of Clinical Investigation, vol. 122, no. 1, pp. 80-90, 2012.

[15] S. Fujii, K. Fujimoto, N. Goto et al., "Characteristic expression of MSX1, MSX2, TBX2 and ENTPD1 in dental pulp cells," Biomedical Reports, vol. 3, no. 4, pp. 566-572, 2015.

[16] X. Li, Q. Cui, C. Kao, G.-J. Wang, and G. Balian, "Lovastatin inhibits adipogenic and stimulates osteogenic differentiation by suppressing PPAR $\gamma 2$ and increasing Cbfa1/Runx 2 expression in bone marrow mesenchymal cell cultures," Bone, vol. 33, no. 4, pp. 652-659, 2003.

[17] F. Ruan, Q. Zheng, and J. Wang, "Mechanisms of bone anabolism regulated by statins," Bioscience Reports, vol. 32, no. 6, pp. 511-519, 2012.

[18] C. Liu, Z. Wu, and H.-C. Sun, "The effect of simvastatin on mRNA expression of transforming growth factor-betal, bone morphogenetic protein-2 and vascular endothelial growth factor in tooth extraction socket," International Journal of Oral Science, vol. 1, no. 2, pp. 90-98, 2009.

[19] Y. Ayukawa, E. Yasukawa, Y. Moriyama et al., "Local application of statin promotes bone repair through the suppression of osteoclasts and the enhancement of osteoblasts at bone-healing sites in rats," Oral Surgery, Oral Medicine, Oral Pathology, Oral Radiology and Endodontology, vol. 107, no. 3, pp. 336-342, 2009.

[20] A. R. Pradeep and M. S. Thorat, "Clinical effect of subgingivally delivered simvastatin in the treatment of patients with chronic periodontitis: a randomized clinical trial," Journal of Periodontology, vol. 81, no. 2, pp. 214-222, 2010.

[21] C.-T. Lee, Y.-T. Lee, H.-Y. Ng et al., "Lack of modulatory effect of simvastatin on indoxyl sulfate-induced activation of cultured endothelial cells," Life Sciences, vol. 90, no. 1-2, pp. 47-53, 2012.

[22] Y. Okamoto, W. Sonoyama, M. Ono et al., "Simvastatin induces the odontogenic differentiation of human dental pulp stem cells 
in vitro and in vivo," Journal of Endodontics, vol. 35, no. 3, pp. 367-372, 2009.

[23] S. Gronthos, M. Mankani, J. Brahim, P. G. Robey, and S. Shi, "Postnatal human dental pulp stem cells (DPSCs) in vitro and in vivo," Proceedings of the National Academy of Sciences of the United States of America, vol. 97, no. 25, pp. 13625-13630, 2000.

[24] R. Karamzadeh, M. B. Eslaminejad, and R. Aflatoonian, "Isolation, characterization and comparative differentiation of human dental pulp stem cells derived from permanent teeth by using two different methods," Journal of Visualized Experiments, no. 69, Article ID 4372, 2012.

[25] J. D. Horton, J. L. Goldstein, and M. S. Brown, "SREBPs: activators of the complete program of cholesterol and fatty acid synthesis in the liver," The Journal of Clinical Investigation, vol. 109, no. 9, pp. 1125-1131, 2002.

[26] X.-Y. Feng, Y.-M. Zhao, W.-J. Wang, and L.-H. Ge, "Msx1 regulates proliferation and differentiation of mouse dental mesenchymal cells in culture," European Journal of Oral Sciences, vol. 121, no. 5, pp. 412-420, 2013.

[27] C. Blin-Wakkach, F. Lezot, S. Ghoul-Mazgar et al., "Endogenous Msxl antisense transcript: in vivo and in vitro evidences, structure, and potential involvement in skeleton development in mammals," Proceedings of the National Academy of Sciences of the United States of America, vol. 98, no. 13, pp. 7336-7341, 2001.

[28] A. E. Coudert, L. Pibouin, B. Vi-Fane et al., "Expression and regulation of the Msxl natural antisense transcript during development," Nucleic Acids Research, vol. 33, no. 16, pp. 52085218, 2005.

[29] S. Petit, F. Meary, L. Pibouin et al., "Autoregulatory loop of Msxl expression involving its antisense transcripts," Journal of Cellular Physiology, vol. 220, no. 2, pp. 303-310, 2009.

[30] S.-L. Cheng, J.-S. Shao, N. Charlton-Kachigian, A. P. Loewy, and D. A. Towler, "MSX2 promotes osteogenesis and suppresses adipogenic differentiation of multipotent mesenchymal progenitors," The Journal of Biological Chemistry, vol. 278, no. 46, pp. 45969-45977, 2003.

[31] F. Ichida, R. Nishimura, K. Hata et al., "Reciprocal roles of Msx2 in regulation of osteoblast and adipocyte differentiation," The Journal of Biological Chemistry, vol. 279, no. 32, pp. 3401534022, 2004.

[32] M. Ishii, A. E. Merrill, Y.-S. Chan et al., "Msx2 and Twist cooperatively control the development of the neural crest-derived skeletogenic mesenchyme of the murine skull vault," Development, vol. 130, no. 24, pp. 6131-6142, 2003.

[33] S.-L. Cheng, A. Behrmann, J.-S. Shao et al., "Targeted reduction of vascular $M s \times 1$ and $M s \times 2$ mitigates arteriosclerotic calcification and aortic stiffness in LDLR-deficient mice fed diabetogenic diets," Diabetes, vol. 63, no. 12, pp. 4326-4337, 2014.

[34] K. M. Catron, H. Zhang, S. C. Marshall, J. A. Inostroza, J. M. Wilson, and C. Abate, "Transcriptional repression by Msx-1 does not require homeodomain DNA-binding sites," Molecular and Cellular Biology, vol. 15, no. 2, pp. 861-871, 1995.

[35] H. Lee, R. Habas, and C. Abate-Shen, "Msxl cooperates with histone H1b for inhibition of transcription and myogenesis," Science, vol. 304, no. 5677, pp. 1675-1678, 2004.

[36] J. Wang and C. Abate-Shen, “The Msx1 homeoprotein recruits G9a methyltransferase to repressed target genes in myoblast cells," PLoS ONE, vol. 7, no. 5, article e37647, 2012.

[37] H. Zhang, G. Hu, H. Wang et al., "Heterodimerization of Msx and Dlx homeoproteins results in functional antagonism,"
Molecular and Cellular Biology, vol. 17, no. 5, pp. 2920-2932, 1997.

[38] M. Zhao, V. Gupta, L. Raj, M. Roussel, and M. Bei, "A network of transcription factors operates during early tooth morphogenesis," Molecular and Cellular Biology, vol. 33, no. 16, pp. 30993112, 2013.

[39] J. Wang, R. M. Kumar, V. J. Biggs et al., “The Msxl homeoprotein recruits polycomb to the nuclear periphery during development," Developmental Cell, vol. 21, no. 3, pp. 575-588, 2011.

[40] Y. Lee, M. J. Schmid, D. B. Marx et al., "The effect of local simvastatin delivery strategies on mandibular bone formation in vivo," Biomaterials, vol. 29, no. 12, pp. 1940-1949, 2008.

[41] C. A. Wassif, P. Zhu, L. Kratz et al., "Biochemical, phenotypic and neurophysiological characterization of a genetic mouse model of RSH/Smith-Lemli-Opitz syndrome," Human Molecular Genetics, vol. 10, no. 6, pp. 555-564, 2001. 

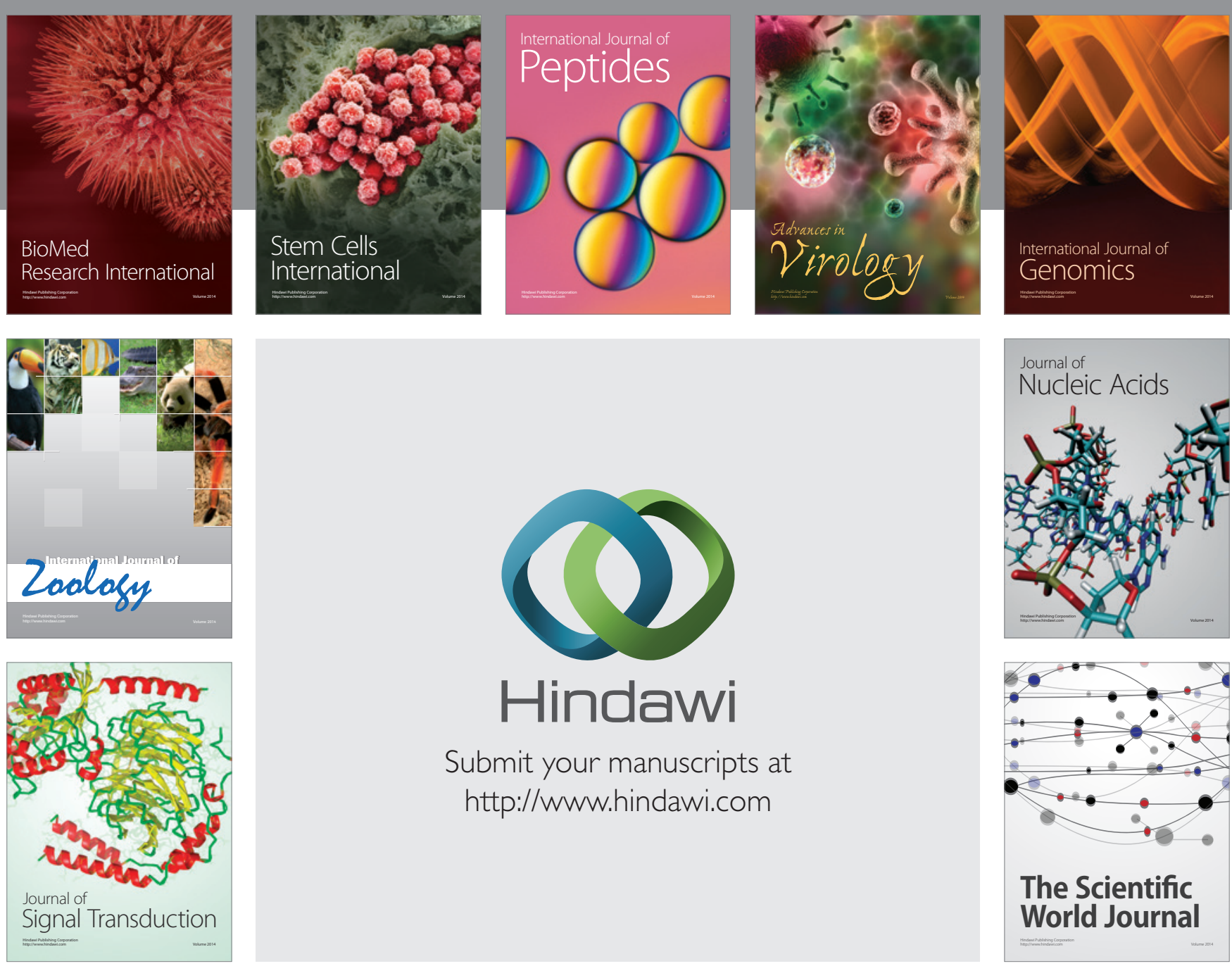

Submit your manuscripts at

http://www.hindawi.com
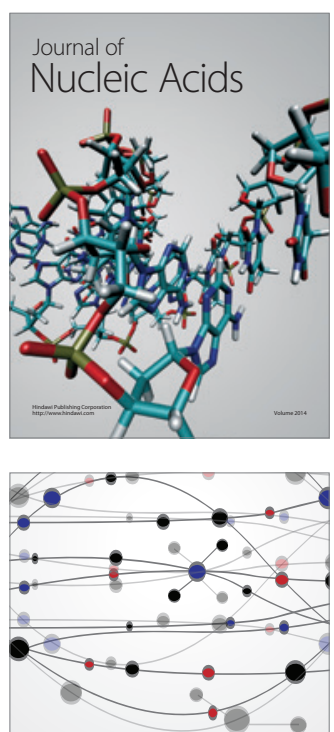

The Scientific World Journal
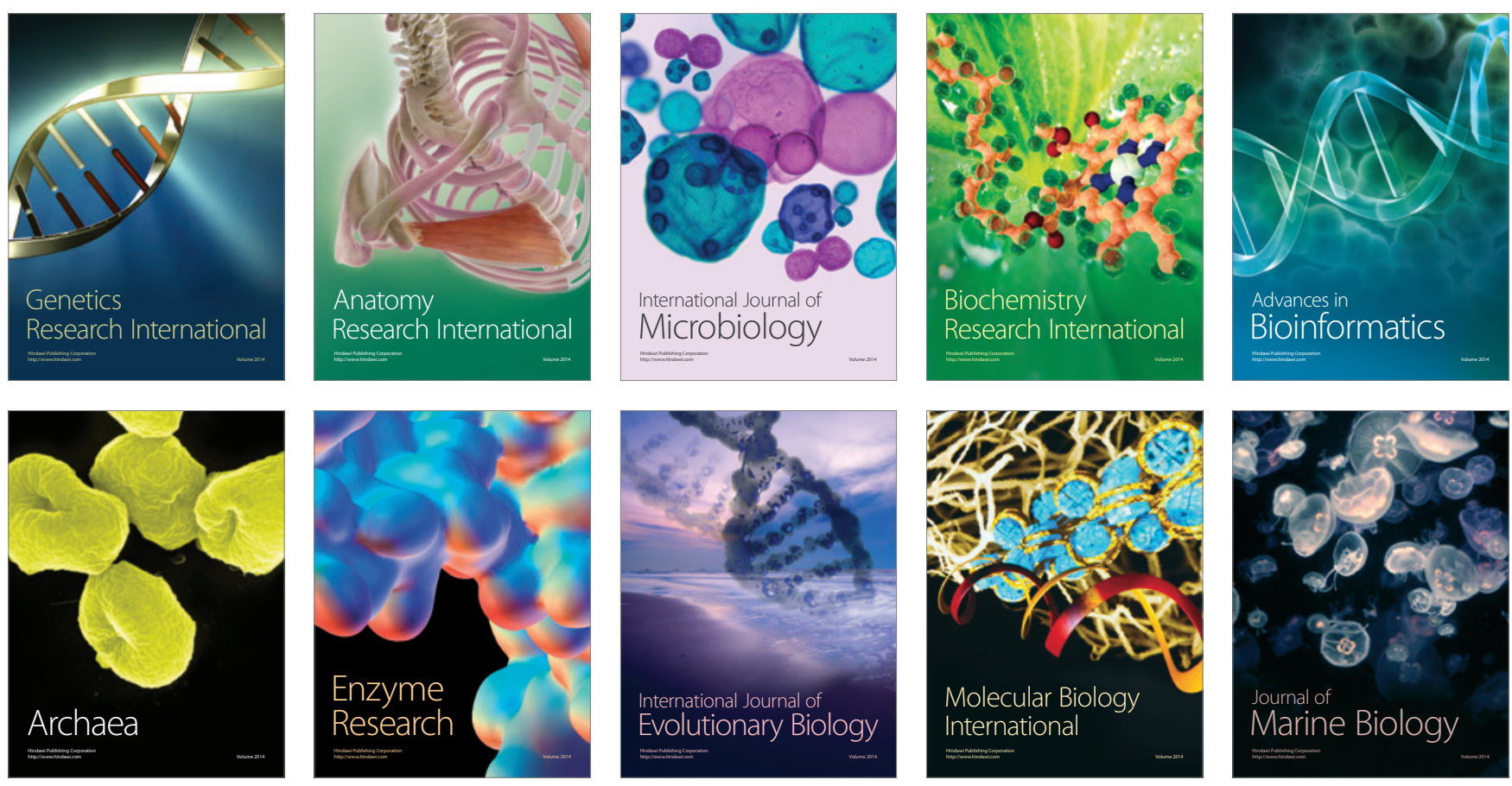